\title{
Magnetoresistive Sensoren für hochdynamische Winkel- und Wegmessung
}

\author{
Dr. Rolf Slatter \\ Sensitec GmbH, Georg-Ohm-Str. 11, 35633 Lahnau \\ Tel.: 06441-9788-13, E-Mail: rolf.slatter@sensitec.com
}

\section{Kurzfassung}

In zahlreichen industriellen und automobilen Anwendungen zeichnet sich ein Trend zu höheren Drehzahlen und schnelleren Linearbewegungen ab. Daraus ergeben sich neue Anforderungen an das Messsystem. Innovative Sensorlösungen werden benötigt, welche hohe dynamische Eigenschaften mit hoher Auflösung, hoher Robustheit und geringen Abmessungen vereinen. Die hohe Bandbreite, die magnetoresistive (MR-) Sensoren bieten, galt bisher eher als „versteckter“ Vorteil dieser Technologie. Das hat sich geändert und inzwischen stellt er in immer mehr Anwendungsbereichen die Basis für die Winkel- und Positionsmessung in Hochgeschwindigkeitsmaschinen dar.

\section{$1 \quad$ Einleitung}

Höhere Geschwindigkeiten bei Dreh- oder Linearbewegungen stellen die Hersteller von Sensoren vor neue Herausforderungen. Der MR-Effekt bietet Maschinenkonstrukteuren und -entwicklern die technologische Grundlage für neuartige Lösungen für schnelle Messaufgaben in einer Vielzahl von Industrien. Magnetische Mikrosysteme basierend auf dem MR-Effekt besitzen schon jetzt eine erhebliche wirtschaftliche Bedeutung entlang der Wertschöpfungskette vom Sensorelement über Module bis hin zu den vielfältigen Anwendungen. Es zeichnet sich ab, dass diese Bedeutung in den kommenden Jahren weiter steigen wird [1].

\section{Trend zu höheren Geschwindigkeiten}

Es gibt verschiedene Gründe für einen Trend zu höheren Geschwindigkeiten in zahlreichen Anwendungen. Wir werden hier nur auf eine Auswahl dieser Gründe eingehen, um den Hintergrund für diese Entwicklung zu erläutern.

Im Bereich rotierender Maschinen, zum Beispiel bei elektrischen Motoren, gilt diese Tendenz sowohl für Industrieantriebe (z. B. Servoantriebe oder Industriepumpen) als auch für andere Anwendungen im Konsumbereich (z. B. Staubsauger). Hauptgrund hierfür ist eine Reduzierung von Volumen und Gewicht bei gleicher Leistung (oder eine Steigerung der Leistung bei konstantem Volumen und Gewicht).

Hinzu kommen verschiedene Anwendungen, die von sich aus eine sehr hohe Geschwindigkeit erfordern, z. B. die neue Generation von elektrisch angetriebenen Turboladern für Automobilanwendungen. In 2010 wurde ein Permanentmagnet-Motor in den Markt eingeführt, welcher eine Rotationsgeschwindigkeit von 1.000.000 1/min erreicht [2], [3].

Die Automobil-Industrie forciert diesen Trend. Die Notwendigkeit, immer strengere Abgasregelungen einzuhalten bzw. eine höhere Effizienz im Kraftstoffverbrauch zu erreichen führt zu der zunehmenden Anwendung von Aufladungstechnologien, wie z. B. Turbolader, für „Downsized Engines“ [4]. Turbolader sind Gaskompressoren, die mehrere schnell drehende Komponenten beinhalten, welche Rotationsgeschwindigkeiten bis 300.000 1/min. aufbringen. Weitere Turbomaschinen, wie zum Beispiel Mikrogasturbinen, werden als Alternative für Batterien bei der mobilen Stromerzeugung vorgeschlagen. Ähnlich verhält es sich mit elektromechanischen Energiespeichern in Form von schnell drehenden Schwungrädern, die jetzt ihren Weg in industrielle und automobile Anwendungen finden.

Der Trend zu höheren Geschwindigkeiten wird ferner durch den Wunsch nach höherer Produktivität begründet. Hochgeschwindigkeitsbearbeitung ist eine inzwischen etablierte Maßnahme, um die Produktivität von komplexen Bauteilen (wie z. B. Turboladerturbinen) zu erhöhen. Der zunehmende Einsatz von Leichtbaukomponenten führt zur Anwendung von neuen Hochgeschwindigkeitsmontagetechniken in der Automobilindustrie, welche wiederum schneller hochauflösender Sensoren für die EchtzeitProzessüberwachung bedarf. 


\begin{tabular}{|c|c|c|c|}
\hline Objekt & & Drehzahl (1/min) & Trend \\
\hline Erde & & 0,000694 & $=$ \\
\hline Uhr (Sekundenzeiger) & & 1 & $=$ \\
\hline Computerfestplatte & & 15.000 & \\
\hline Formel 1 Rennmotor & & 20.000 & \\
\hline Hochfrequenzmaschinenspindel & & 100.000 & \\
\hline Mikrogasturbine & & 100.000 & \\
\hline Energiespeicher (Schwungrad) & & 200.000 & \\
\hline Turbomolekularpumpe & & 200.000 & \\
\hline Turbolader & & 3.00 .000 & \\
\hline Dentalbohrer & & 4.00 .000 & \\
\hline $\begin{array}{l}\text { Ultra-Hochgeschwindigkeits- } \\
\text { permanentmagnet-Motor }\end{array}$ & & 1.000 .000 & \\
\hline
\end{tabular}

Tabelle 1: Drehgeschwindigkeiten einiger bekannter Objekte und Hochgeschwindigkeitsmaschinen 
Ähnliche Entwicklungen zeichnen sich im Bereich der Antriebstechnik ab. Linearmotoren in Werkzeugmaschinen erreichen inzwischen Lineargeschwindigkeiten bis zu $5,5 \mathrm{~m} / \mathrm{s}$ und Beschleunigungen bis zu $30 \mathrm{~g}$. Hydraulikzylinder für Hochleistungs-Textilmaschinen erreichen sogar Lineargeschwindigkeiten von mehr als $30 \mathrm{~m} / \mathrm{s}$ und Beschleunigungen bis zu $850 \mathrm{~g}$.

Und dieser Trend scheint sich fortzusetzen, wie in der rechten Spalte von Tabelle 1 dargestellt, die zeigt, dass für die meisten Objekte, die hier aufgeführt sind, in Zukunft noch höhere Geschwindigkeiten zu erwarten sind.

\section{Vorteile von MR Sensoren in Hochgeschwindigkeitsanwendungen}

Aufgrund der größeren Geschwindigkeiten sind völlig neue Anforderungen zu erfüllen, die innovative Sensorlösungen erfordern. Sensoren spielen in mehreren verschiedenen Phasen innerhalb der Produktlebenszyklen eines Hochgeschwindigkeitsprodukts eine wichtige Rolle:

- Produkt- und/oder Komponentendesign und -entwicklung

- Produkttest

- Produktherstellung (z. B. während der Bearbeitung und Montage)

- Produkteinsatz, Wartung und Reparatur (z. B. Zustandsüberwachung)

Die Anforderungen, die dabei an den Sensor gestellt werden, können in jeder Phase sehr anspruchsvoll sein. Die Notwendigkeit hochdynamische Leistungen zu erzielen und dabei ein berührungsloses Messverfahren anzuwenden, liegt auf der Hand. In vielen Hochgeschwindigkeitsanwendungen kommen jedoch noch weitere Forderungen hinzu wie hohe Auflösung, hohe Robustheit sowie miniaturisierte Abmessungen.

Die Vorteile von MR Sensoren in konventionellen Industrieanwendungen sind im Wesentlichen bekannt. Die extrem hohe Bandweite von MR Sensoren gilt jedoch bis vor kurzem als seine Art "verborgener" Vorteil. MR Sensoren sind in der Lage, konstante als auch wechselnde Magnetfelder zu messen. Darüber hinaus kann das alternierende Magnetfeld mit einer sehr hohen Frequenz gemessen werden - die Literatur verweist auf das erfolgreiche Messen alternierender Magnetfelder mit einer Frequenz von $100 \mathrm{MHz}$ [5] oder sogar $300 \mathrm{MHz}$ [6]. Die Frequenzbandbreite von Magnetisierungsumkehrungen in Dünnfilmen geht theoretisch von Gleichstrom zum Gigahertz-Bereich. Messungen bei Sensitec am im Kapitel 6 vorgestellten Zahnsensormodul beweisen, dass eine Frequenz von $10 \mathrm{MHz}$ ohne einen wesentlichen Verlust hinsichtlich Genauigkeit oder Auflösung erreicht werden kann. Das entspricht einer theoretischen linearen Auflösung von $10 \mu \mathrm{m}$ bei einer Lineargeschwindigkeit von $100 \mathrm{~m} / \mathrm{s}$ oder einer Winkelauflösung von $0,06^{\circ}$ bei einer Drehzahl von $100.0001 / \mathrm{min}$. Es sollte noch darauf hingewiesen werden, dass die maximale gemessene Frequenz lediglich durch die verfügbaren Prüfgeräte begrenzt wurde und nicht durch das Sensorelement selbst.

Im Vergleich zu induktiven Sensoren besteht der entscheidende Vorteil darin, dass MR Sensoren das Magnetfeld H (oder die Magnetflussdichte B) direkt messen, während induktive Sensoren die derivative $\mathrm{dB} / \mathrm{dt}$ messen. Daher benötigt der MR Sensor keinen integrierten Schaltkreis, durch den sich zusätzliche Fehler einschleichen. Die Empfindlichkeit des MR Sensors ist nicht abhängig von der Geschwindigkeit des Induktionsstromwechsels, was zu Problemen führen kann, wenn Informationen von bewegten Quellen ausgelesen werden.

Wie an späterer Stelle noch anhand von Anwendungsbeispielen demonstriert wird, MR Sensoren können häufig unter Betriebsbedingungen eingesetzt werden, denen optische Sensoren nicht standhalten können, wie z. B. hohe Temperaturen oder in Umgebungen, die durch Verschmutzung aufgrund von Öl, Fett, Staub oder Wasser belastet werden.

Darüber hinaus eignen sich MR Sensoren hervorragend zur so genannten „passiven“ Messung, bei der ein existierendes ferromagnetisches Maschinenelement als Maßverkörperung benutzt wird. Diese Eigenschaft spielt eine wichtige Rolle in Hochgeschwindigkeitsanwendungen, wo die Nutzung eines magnetischen Objekts (oder einer optischen Encoderscheibe) oftmals durch die hohe mechanische Last, die auf die Maßverkörperung wirkt, unmöglich wird.

Da MR Sensoren das präzise Messen von Weg oder Winkel bei der Entwicklung von Komponenten für Hochgeschwindigkeitsanwendungen ermöglichen, bieten sie dem Nutzer signifikante Vorteile: 
- Marktgerechte Produktentwicklung

- Schnellere Marktreife

- Schnellere Stückzahlerhöhung und Einhaltung der Zielkosten

- Erhöhen der Gewinnspannen and Reduzierung der Lieferrisiken

- Reduzierung der Service-, Garantie- und Wartungskosten

\section{Grundlagen magnetoresistiver Sensortechnologie}

Der magnetoresistive (MR-) Effekt ist seit über 150 Jahren bekannt. In 1857 entdeckte der britische Physiker William Thomson, später Lord Kelvin, dass sich der elektrische Widerstand eines stromdurchflossenen Leiters unter dem Einfluss eines Magnetfeldes verändert. Die sensorische Nutzung dieses Effekts konnte jedoch erst vor ca. 30 Jahren mit der Weiterentwicklung der Dünnschichttechnik industriell umgesetzt werden. Durch eine geschickte Anordnung der Strukturen innerhalb des Sensors können die unterschiedlichsten Sensoren konstruiert werden, um Magnetfeldwinkel, -stärke oder -gradienten zu erfassen. Der von Thomson entdeckte Effekt wurde als „Anisotroper magnetoresistiver Effekt" (AMR) benannt und wies eine Widerstandsänderung von nur wenigen Prozent auf. Trotzdem konnte dieser Effekt erfolgreich in Schreib-Leseköpfen für Computer-Festplatten millionenfach umgesetzt werden. Ende der 80er Jahre wurde der Giant magnetoresistive Effekt (GMR) von Prof. Grünberg am Forschungszentrum Jülich und Prof. Fert an der Universität Paris entdeckt. Hier werden Widerstandsänderungen von über 50 \% gemessen, welche noch weitere Anwendungsbereiche für MR-Sensoren eröffneten. Diese Entdeckung wurde in 2007 mit dem Nobelpreis für Physik ausgezeichnet.

Inzwischen werden die Leseköpfe moderner Festplattenlaufwerke fast ausschließlich mit magnetischen Tunnelkontakten auf Basis des Tunnel magnetoresistive Effekts (TMR) realisiert. Diese Technologie verfügt über neue Eigenschaften, die außer in der Speichertechnologie auch für spezielle Sensoranwendungen interessant sind. So zeigen TMR-Sensoren eine sehr hohe Empfindlichkeit bei sehr geringem Stromverbrauch. AMR- und GMR-Sensoren werden bei Sensitec in Hessen und Rheinland-Pfalz in Serie gefertigt. Die ersten TMR-Sensoren stehen kurz vor der Markteinführung [7].

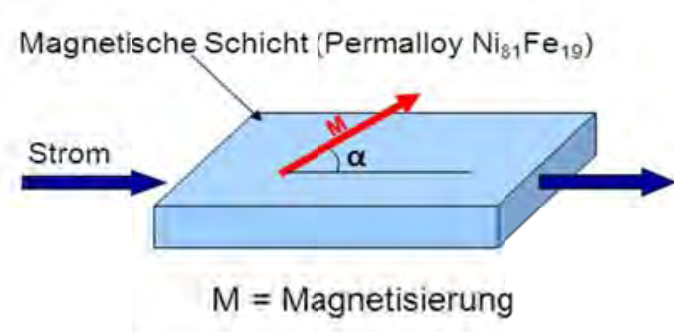

a) AMR Streifen

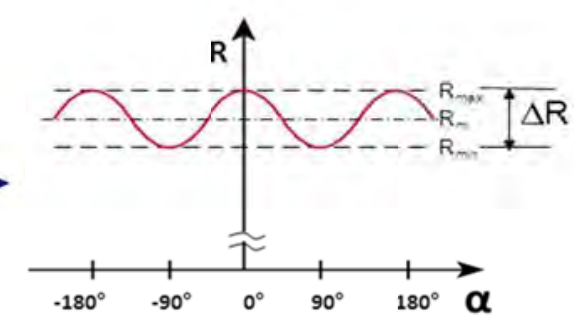

b) Widerstandsveränderung

Abb. 1 AMR-Prinzip

Der anisotrope magnetoresistive Effekt (AMR-Effekt) ist in ferromagnetischen Materialien wie Eisen, Nickel und Kobalt beobachtbar. Der spezifische Widerstand $\rho$ dieser Materialien ist abhängig vom Winkel $\varphi$ zwischen Strom I und Magnetisierung M. Sind die Richtungen von Strom und Magnetisierung parallel, ist der Widerstand $\rho$ maximal, und stehen die Richtungen von Strom und Magnetisierung senkrecht aufeinander, ist $\rho$ minimal. Fließt der Strom in Streifenlängsrichtung, kann der spezifische Widerstand $\rho$ durch den Widerstand R ersetzt werden und der AMR-Effekt kann dann durch folgende Gleichung beschrieben werden:

$$
R(\alpha)=R_{m}+\frac{\Delta R}{2} \cos (2 \alpha)
$$

Der Verlauf der Funktion $R(\alpha)$ für $0^{\circ}<=\alpha<=360^{\circ}$ ist in Abb. $1 \mathrm{~b}$ dargestellt. Man erkennt, dass sich der Widerstand $\mathrm{R}$ mit dem doppelten Winkel $2 \alpha$ um einen mittleren Widerstand $R_{m}$ ändert. 
Der Aufbau eines AMR Winkelsensors ist verhältnismäßig einfach (Abb. 2). Deswegen sind die passiven Widerstandselemente von Grund aus sehr zuverlässig. Auf einem Silizium-Wafer befindet sich eine Siliziumoxidschicht, die eine Isolation zwischen Wafer und MR-Schicht bildet. Darauf befindet sich die MRSchicht aus einer Nickel-Eisen-Legierung. Um optimale Sensoreigenschaften zu erreichen, wird Permalloy $\left(\mathrm{Ni}_{81} \mathrm{Fe}_{19}\right)$ eingesetzt, welches einen hohen Widerstandswert und sehr geringe Magnetostriktion aufweist.

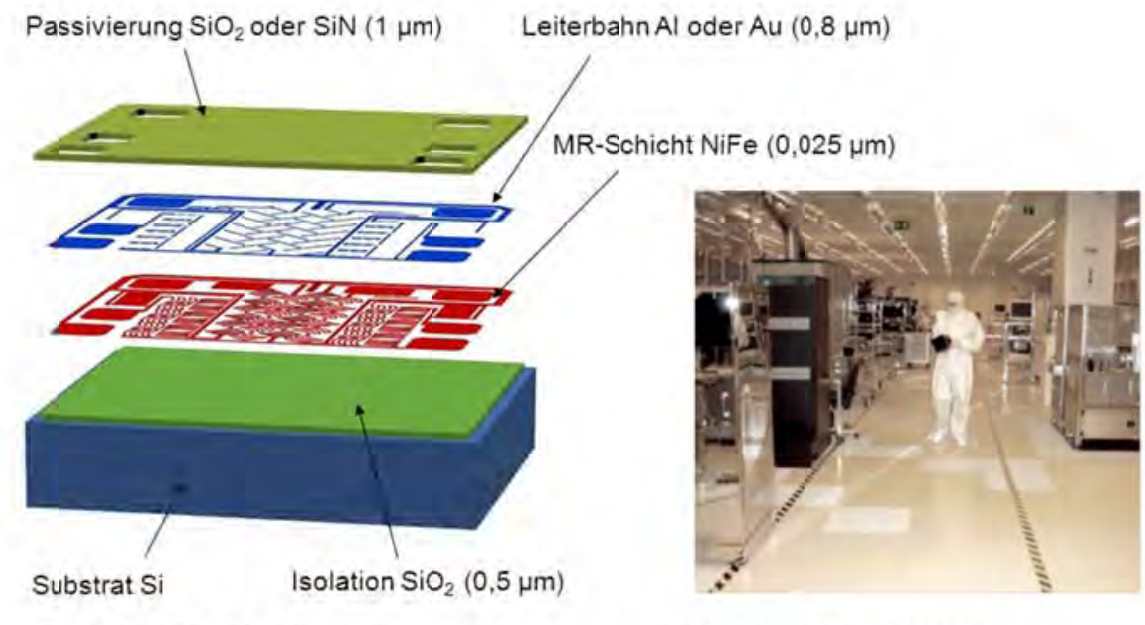

a) Aufbau eines AMR Sensors

b) Reinraum für Lithographie

Abb. 2 Aufbau eines AMR Winkelsensors

Als nächstes wird eine Schicht aus Gold oder Aluminium mit den Sensorleitbahnen und Kontakten aufgedampft oder gesputtert. Abschließend wird eine Schicht aus Siliziumnitrid aufgebracht, welche als Passivierung gegenüber Umwelteinflüsse dient. Moderne Fertigungstechniken in der Waferproduktion und geeignete Materialpaarungen erlauben es, die Temperaturkoeffizienten für die Ausgangssignalamplitude, die Offsetspannung und den Sensorwiderstand auf ein Minimum zu reduzieren.

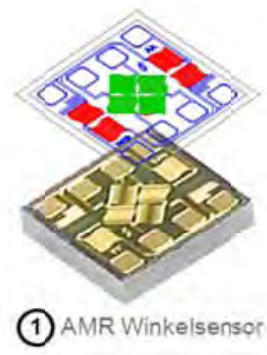

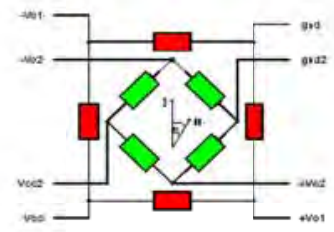

(2) Wheatstone-Brücke (Sinus und Cosinus)

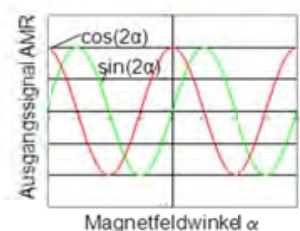

(3) Ausgangssignaleiner AMR Brücke

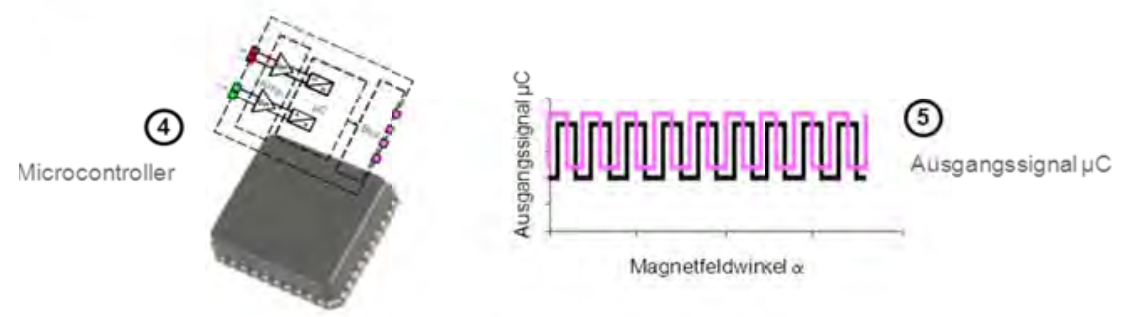

Abb. 3 Entstehung und Verarbeitung des Sensorsignals

Um Temperatureinflüsse auf dem Sensorchip weiter zu minimieren, werden üblicherweise vier Widerstände zu einer Wheatstone'schen Brücke verschaltet. In AMR Winkelsensoren erzeugen zwei um $45^{\circ}$ zueinander gedrehte Wheatstone'sche Brücken jeweils ein Sinus- und ein Kosinus-Signal. Jede besteht aus mäanderförmigen Widerstandsblöcken, die auf das äußere Magnetfeld mit einer resistiven Änderung reagieren. 
Abb. 3 zeigt die Signalkette eines AMR Winkelsensors [8]. In einem beispielhaften, einfachen Einsatzfall wird ein diametral magnetisierter Magnet auf einem drehenden Wellenende angebracht. Der Winkelsensor wird auf der Rotationsachse in einem Abstand von einigen Millimetern vom Magnet befestigt. Das rotierende Magnetfeld erzeugt in der gleichen Ebene wie die Wheatstone-Brücke zwei Ausgangssignale, die die doppelte Frequenz des Winkels $\alpha$ zwischen Sensor und Magnetfeldrichtung aufweisen. Ein Ausgangssignal repräsentiert die $\sin (2 \alpha)$ Funktion, während das andere Signal die $\cos (2 \alpha)$ Funktion gemäß Gleichung 1 darstellt. Diese Signalform erlaubt die Absolutmessung der Winkel bis $180^{\circ}$ und ermöglicht zudem eine Selbstdiagnose in sicherheitskritischen Anwendungen mittels der Gleichung $\left(\sin ^{2} \alpha+\right.$ $\left.\cos ^{2} \alpha\right)=1$.

Eine Verstärkerschaltung wird häufig zur Verstärkung der rohen Sensorsignale eingesetzt. Diese wird entweder als komplette integrierte Schaltung oder über eine Kombination aus einzelnen Komponenten und integrierten Schaltkreisen, wie z. B. Operationsverstärker, implementiert. Diese Signale können dann mittels Interpolations ASIC (Application Specific Integrated Circuit), ASSP (Application Specific Standard Product) oder Mikrocontroller digitalisiert werden, um das digitale Ausgangssignall bereitzustellen.

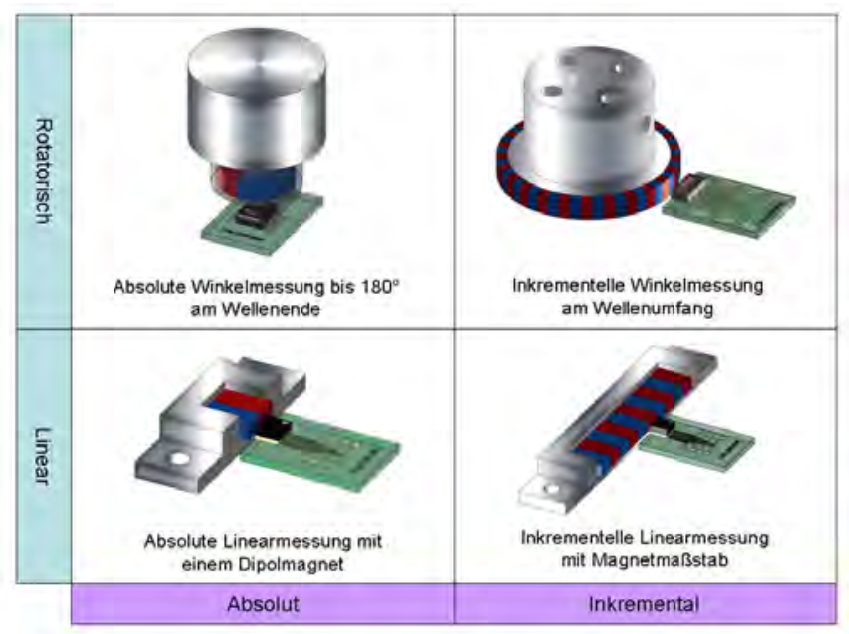

Abb. 4 Messanordnungen für Weg- und Winkelmessung

In Abb. 4 sind verschiedene Messanordnungen aufgezeigt, die sich verwirklichen lassen. Dabei erkennt man, dass es sich nicht nur um die Erfassung von rotatorischen Bewegungen, sondern auch um lineare Bewegungen handeln kann. Des Weiteren sind sowohl inkrementelle als auch absolute Winkel- und Längenmessaufgaben lösbar. Für eine detaillierte Erläuterung der komplexeren GMR- und TMR-Effekte, die in Schichtsystemen mit mindestens zwei ferromagnetischen Schichten und einer nichtmagnetischen, metallischen Zwischenschicht (GMR) oder Isolationsschicht (TMR) auftreten, wird auf die Literatur hingewiesen [5], [6]. Abb. 5 zeigt einen schematischen Vergleich der Sensorelemente und deren Verhalten. In der Sensorik sind AMR, GMR und TMR komplementäre Technologien. Alle drei MR-Effekte verfügen über spezifische Vorteile, die in einer bestimmten Anwendung entscheidend sein können.

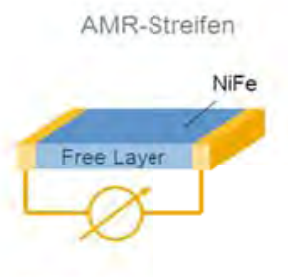

Barber-Pole Characteristic

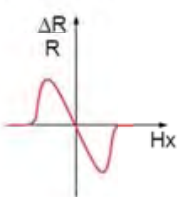

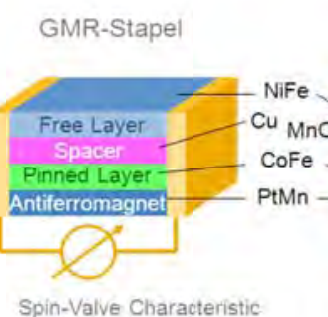

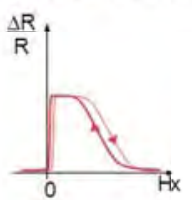

TMR-Stapel
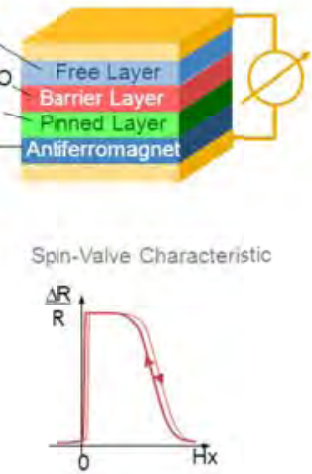

Abb. 5 Vergleich der XMR-Technologien 


\section{$5 \quad$ Vorteile und Nutzen von MR-Sensoren}

Die verschiedenen MR-Effekte verfügen gemeinsam über eine Reihe von Vorteilen, die alle dazu beigetragen haben, dass sich MR Sensorik als richtige Wahl in den anschließend beschriebenen Anwendungen erwiesen hat [8], [9]:

- Hohe Auflösung und hohe Genauigkeit

- Hohe Dynamik mit einer Bandbreite bis über $10 \mathrm{MHz}$

- Sehr robust mit hoher Unempfindlichkeit gegenüber Öl, Schmutz und sehr hohe oder sehr niedrige Umgebungstemperaturen

- Hohe Zuverlässigkeit

- Extrem kleine Abmessungen und sehr niedrige Leistungsaufnahme

- Wartungs- und verschleißfreier Betrieb aufgrund von berührungslosem Messen

Diese Merkmale bieten dem Anwender folgenden Nutzen:

- Großer Arbeitsabstand zwischen Sensor und zu messendem Objekt, wodurch sich der Konstruktions- und Montageaufwand reduziert.

- Die hohe Empfindlichkeit bietet die Möglichkeit durch eine Gehäusewand zu messen.

- Höchst präzise Winkel- und Linearmessung mit hoher Auflösung für eine exakte Messleistung bei hoher Positioniergenauigkeit.

- Hohe Bandbreite, um sehr dynamische Prozesse zu überwachen [6].

- Zuverlässigkeit und Sicherheit unter schwierigen Betriebsbedingungen.

- Kompaktes, leichtes Messsystem, welches Messungen an schwer zugänglichen Stellen ermöglicht.

- Lange Lebensdauer durch verschleißfreien Betrieb.

MR Sensoren weisen viele Vorteile gegenüber potentiometrischen, induktiven und optischen Sensoren [10] auf.

\section{Anwendungsbeispiele}

Es gibt mehrere Gründe für den oben erwähnten Trend zu höheren Geschwindigkeiten. Bei elektrischen Motoren, sowohl in Industrie- (z. B. Servoantriebe oder Pumpenantriebe) als auch in KonsumAnwendungen (z. B. in Staubsaugern) wird eine immer höhere Leistungsdichte angestrebt. Es werden entweder Bauvolumen und Gewicht bei gleichbleibender Leistungsaufnahme reduziert, oder eine erhöhte Leistung beim gleichen Bauvolumen abgegeben. In beiden Fällen trägt eine erhöhte Motorgeschwindigkeit dazu bei. Es gibt natürlich auch Anwendungen, wo intrinsisch eine hohe Geschwindigkeit erforderlich ist, wie z. B. bei Turbomaschinen. Nicht zuletzt führen höhere Bearbeitungsgeschwindigkeiten zu einer bemerkenswerten Steigerung der Produktivität und Werkstückqualität in der spanenden Bearbeitung. Dieser Trend führt teilweise zu komplett neuen Aufgabenstellungen, die innovative Sensorlösungen benötigen.

\subsection{Winkelmessung in Auswuchtmaschinen}

Auswuchten ist ein Vorgang, bei dem die Masseverteilung eines Rotors geprüft und soweit verbessert wird, dass die unwuchtbedingten Schwingungen und Kräfte in zulässigen Grenzen liegen. Als Rotor in diesem Sinne gelten nicht nur alle die Teile, die sich im Betriebzustand drehen, sondern auch jene, die aus funktionalen Gründen drehbar gelagert sind. Damit ist dieser Vorgang von besonderer Bedeutung für die Antriebstechnik. Inzwischen wird bei nahezu allen Rotoren das Auswuchten als unbedingt notwendig angesehen, sei es, um die Lebensdauer der Maschine zu verlängern, ihre Funktion zu verbessern oder um einen schwingungsarmen Lauf zu gewährleisten.

Es gibt auch ständig neue Herausforderungen, entweder in Form von neuen Produkten mit einer besonderen Aufgabenstellung, wie z. B die Auswuchtung von Turboladern, die Messdrehzahlen bis zu 200.000 $1 /$ min erreichen, oder auch in einer steigenden Erwartungshaltung hinsichtlich eines besseren Auswuchtergebnisses für bekannte Produkte, wie z. B. Rotoren für Elektromotoren in der Automatisierungstechnik. Bei gleichzeitig hoher Auswuchtgüte erwarten Anwender weiterhin eine erhöhte Betriebssicherheit bei verkürzten Taktzeiten. Diese Kombination an Anforderungen stellt die Entwickler von Auswuchtmaschinen (Abb. 6) vor neuen Aufgaben, die mit bisherigen Maschinenkonzepten nicht zu erfüllen sind. 


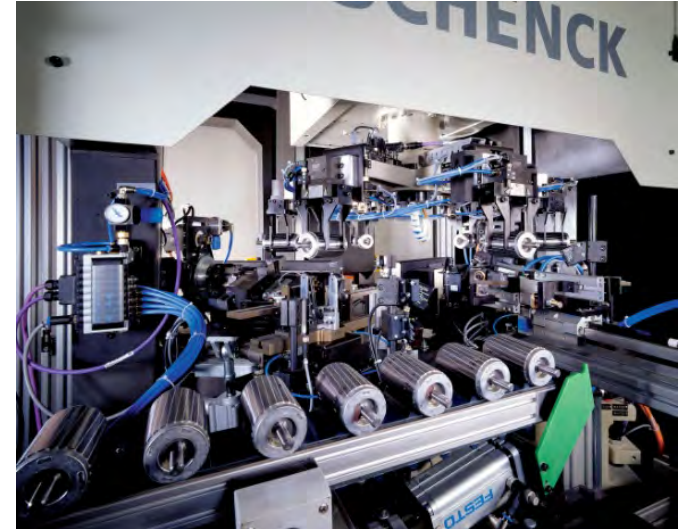

Abb. 6 Auswuchtmaschine (Bildquelle: Schenck Rotec $\mathrm{GmbH}$ )

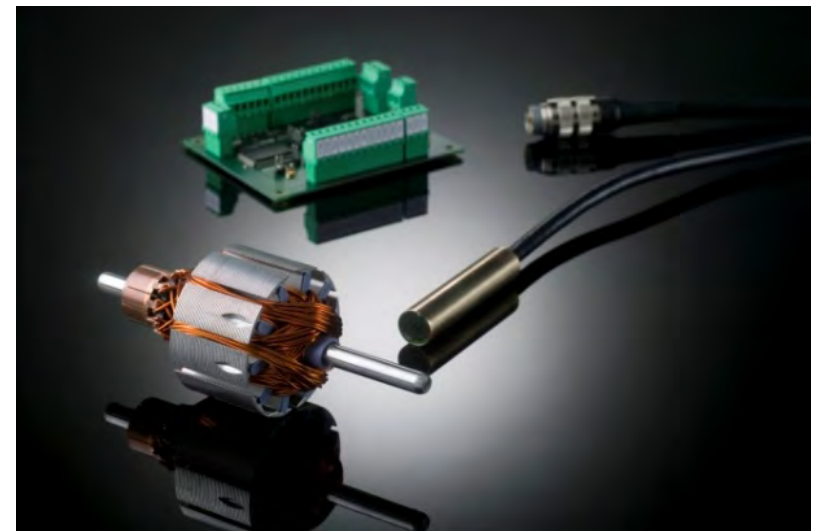

Abb. 7 Sensorkopf, Elektronik und ausgewuchtete Motorwelle

Der Lösung liegt in dem Aufmagnetisieren der Rotorwelle durch Anbringen einer magnetischen Marke (Abb. 8). Damit wird der Rotor zum Bestandteil des Inkrementalgebers und beseitigt so Probleme durch Schlupf. Bei der bisherigen Lösung war der Inkrementalgeber mittels Riemen angetrieben, was die zulässige Rotationsgeschwindigkeit stark begrenzte. Verschiedene FEM-basierte Feldberechungen wurden unternommen, um ein besseres Verständnis der Vorgänge beim Magnetisieren zu bekommen. Versuchsreihen und erste Messungen mit MR-basierten Sensorprototypen zeigten, dass eine Abtastung in der gewünschten Art und Weise möglich war [6].

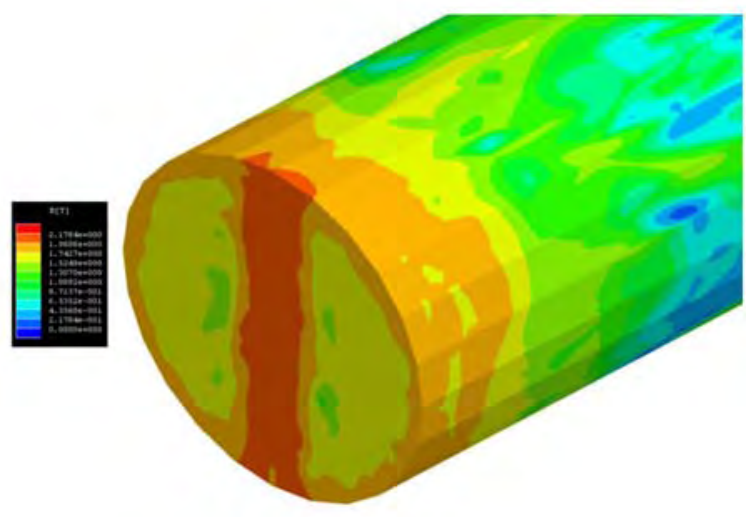

Abb. 8 Aufmagnetisierte Rotorwelle

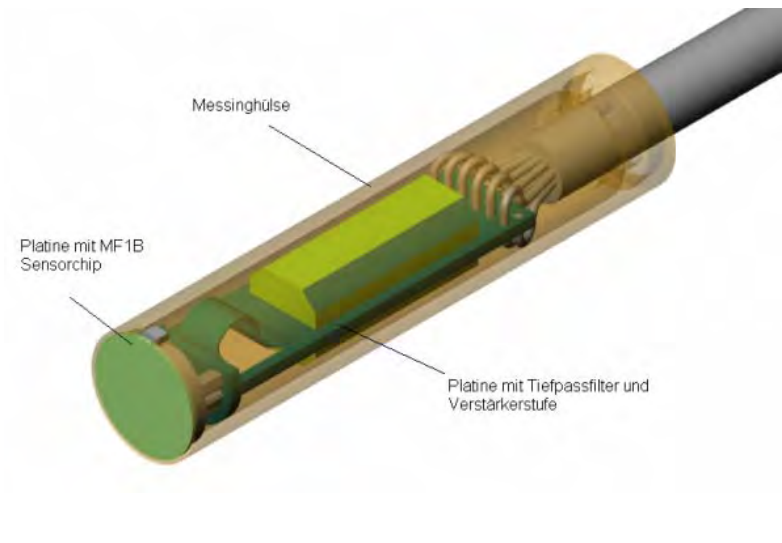

Abb. 9 Aufbau des Sensorkopfes (Bildquelle: Schenk Rotec $\mathrm{GmbH}$ )

Die Welle mit ihrem rotierenden, magnetischen Dipol wird abgetastet durch den Sensorkopf, und der Unwuchtwinkel wird durch den prozessorgesteuerten Digitalteil des Sensorsystems generiert. Das neue Sensorsystem besteht aus 3 Modulen (Abb. 7):

a) Eine Magnetisiereinheit zum Aufbringen des magnetischen Dipols auf der Rotorachse

b) Ein analoger Sensorkopf, um den rotierenden Dipol abzutasten und

c) eine digitale Signalverarbeitungseinheit mit Interpolations-ASIC und Prozessorsteuerung, um Positions- und Drehzahlinformation an die übergeordnete Steuerung abzuliefern.

Im Sensorkopf ist ein AMR-Magnetfeldsensor vom Typ MF1B integriert sowie ein Tiefpassfilter und Verstärkerstufe (siehe Abb. 8). Dieser miniaturisierte Sensorchip beinhaltet eine Wheatstone-Brücke und eine Flipspule, um die Offsetstabilität und Empfindlichkeit des Sensors zu maximieren. Der Sensorkopf liefert Sinus- und Kosinus-Signale sowie komplementäre Signale, die in der digitalen Signalverarbeitungseinheit weiter prozessiert werden. Das neue System ist um den Faktor 1000 empfindlicher als alle bisher bekannten Systeme am Markt. Diese hohe Empfindlichkeit ermöglicht eine Winkelgenauigkeit besser als $1 \mathrm{Grad}$ und große Arbeitsabstände zwischen 1 und $7 \mathrm{~mm}$ [9]. Last, but not least, das neue Konzept öffnet ganz neue Möglichkeiten. Das Auswuchten von Turboladern mit Messdrehzahlen bis 200.000 1/min ist 
inzwischen möglich gemacht wurden. In Tests an einer mit Pressluft angetriebenen Turbine für einen Zahnarztbohrer wurden sogar Drehzahlen bis 420.000 1/min erfolgreich abgetastet.

\subsection{Winkelmessung in hochdrehenden, luftgelagerten Spindeln}

Eine quasi-dauerhafte Aufmagnetisierung eines Bauteils oder die Anbringung eines zusätzlichen Magnets als Maßverkörperung ist nicht immer möglich. In diesem Fall wird die periodische Modulierung eines starken Magnetfeldes durch ferromagnetische Funktionsbauteile (Zahnräder oder Zylinderstangen) zur berührungslosen und dynamischen Erfassung von Bewegungen genutzt. Abb. 11 zeigt die Simulation des Feldverlaufes einer durch einen Stützmagnet aufmagnetisierten weichmagnetischen Zahnstruktur. Eine Modulation der Feldstärke ist klar ersichtlich. Der eingesetzte Zahnsensor ist ein Gradientensensor oder Magnetfelddifferenzsensor. Er misst die Differenz der Feldstärke an zwei verschiedenen Punkten. Der Sensor ist aufgebaut aus zwei Wheatstone-Brücken (Vollbrücken), die geometrisch zueinander um ein Viertel der Periodenlänge $\lambda$ der Maßverkörperung versetzt sind. Die Einzelwiderstände der WheatstoneBrücke bestehen wiederum aus mehreren GMR-Streifen, die ineinander verschachtelt sind. In einem optimal aufeinander abgestimmten Gehäuse $(13 \times 5,5 \times 3,5 \mathrm{~mm})$ werden sowohl der GMR Zahnsensorchip als auch der Stützmagnet untergebracht und gegen Fremdeinflüsse geschützt (Abb. 11). Der starke Magnet erzeugt eine sehr hohe Feldstärke, was die Störempfindlichkeit dieses Moduls deutlich reduziert und ein sehr gutes Signal-Rausch-Verhalten gewährleistet. Die GLM Zahnsensormodule (Abb. 10) sind in verschiedenen Ausführungen erhältlich, um die einfache Nutzung mit unterschiedlichen Zahnteilungen (1, 2 und $3 \mathrm{~mm}$ ) oder Zahnmodulen $(0,3$ und 0,5$)$ zu ermöglichen.

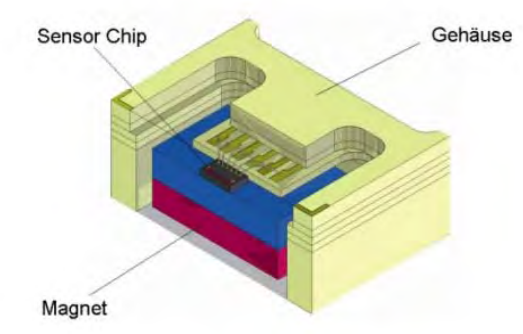

Abb. 10 GLM Zahnsensormodul

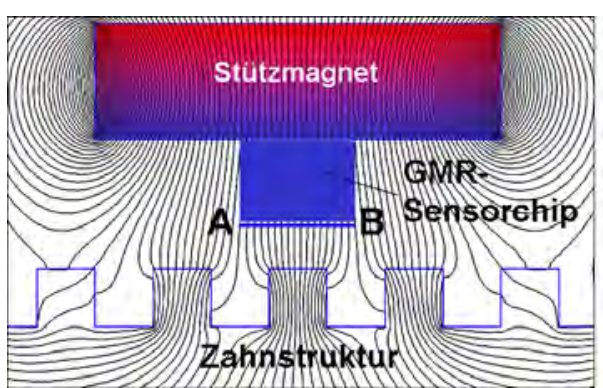

Abb. 11 Funktionsprinzip

Die Module können bei Umgebungstemperaturen zwischen -40 und $+125{ }^{\circ} \mathrm{C}$ eingesetzt werden und liefern ein klirrarmes Sinus-/Kosinus-Signal mit hohem Signal-Rausch-Verhältnis. Die hohe Signalgüte macht eine sehr präzise 100 fache Interpolation sinnvoll, welche lineare Geschwindigkeiten von mehr als $50 \mathrm{~m} / \mathrm{s}$ bei einer Auflösung von $10 \mu \mathrm{m}$ oder die Erfassung von Drehzahlen von mehr als 100.000 1/min bei Auflösungen im Winkelminutenbereich zulässt. Die GLM Zahnsensormodule ermöglichen dadurch eine präzise und hochdynamische Erfassung von linearen und rotatorischen Bewegungen und werden in Gebersystemen für geregelte hydraulische, pneumatische und elektrische Antriebe im Maschinen- und Anlagenbau eingesetzt. Eine sehr aktuelle Anwendung bildet die Winkelmessung in hochdrehenden, luftgelagerten Spindeln.

Überall dort, wo hohe Anforderungen an Werkstückmaßhaltigkeit, Oberflächenqualität und Zerspanleistung gestellt werden, insbesondere in der Feinbearbeitung oder im Werkzeug- und Formenbau, ist die Hochgeschwindigkeitsbearbeitung nicht mehr wegzudenken. Aufgrund erheblich gesteigerter Werkzeugumdrehungsfrequenzen und damit auch Vorschubgeschwindigkeiten werden bessere Oberflächengüten und eine raschere Bearbeitung ermöglicht. Durch die Verwendung von GLM-Sensoren werden gerade in der Luftlagertechnik einige entscheidende Vorteile generiert. So kann die für die Kommutierung des Motors und die Positionsregelung der Welle notwendige Teilung mit einem Modul von 0,3 mm direkt auf der Welle in Form von radialen Bohrungen verwirklicht werden. Dadurch kann auf Encoderscheiben oder ähnliches verzichtet werden und die Unwucht der Welle wird auf ein Minimum reduziert. Durch die Möglichkeit, zwei magnetisch gleichartige Sensoren gegenüberliegend zu platzieren, wird die ohnehin schon sehr geringe Magnetkraft der Sensoren kompensiert. Damit wird die axiale und radiale Verlagerung der Spindelwelle durch die Sensorik auf unter $10 \mathrm{~nm}$ reduziert. 


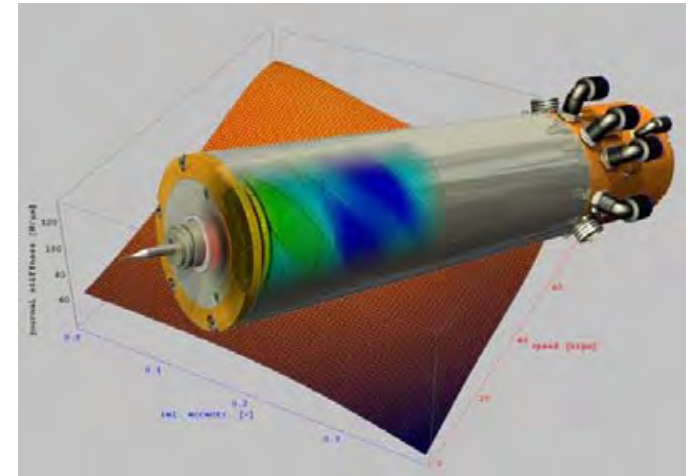

Abb. 12 Luftgelagerte Motorspindel (Bildquelle: Levicron $\mathrm{GmbH}$ )

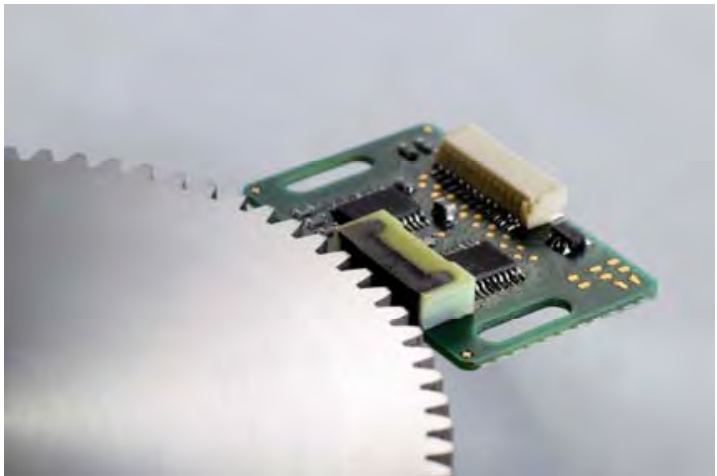

Abb. 13 GLM Zahnsensormodul mit Zahnrad als Maßverkörperung

Durch die GLM Sensorik ist es möglich, eine luftgelagerte Industriespindel mit SK 25 Werkzeugspannsystem und $100 \mathrm{~mm}$ Klemmdurchmesser bei Drehzahlen bis zu $90.000 \mathrm{U} / \mathrm{min}$ und einer Auflösung von 0,1 ${ }^{\circ}$ zu positionieren und kommutieren (Abb. 12). Mit der ersten luftgelagerten Spindel mit HSK Werkzeugspannsystem wurde die Basis für eine industrielle Motorspindel gelegt. Hier wird dem Wunsch der Kunden aus dem Markt der Ultrapräzisionsbearbeitung entsprochen, eine robuste, bedienungsfreundliche ultrapräzise Luftlager-Motorspindel zu entwickeln, die zudem zahlreiche ebenfalls kundengetriebene Innovationen enthält. Hierzu zählen die aktive Kühlung, ein automatisches Spannzangensystem zur Werkzeugklemmung sowie die robuste Lagerung.

\subsection{Ventilhubmessung im befeuerten Motor}

Die o. g. Anwendungsbeispiele zeigen einen ganz neuen Ansatz auf - d. h. die direkte Messung von Winkeln oder Wegen an vorhandenen ferromagnetischen Bauteilen. Dieses Prinzip macht Messungen an Zahnrädern, Zahnstangen, Wellen oder Gewindespindeln möglich, ohne die Notwendigkeit einer separaten magnetischen Maßverkörperung in Form eines Dipolmagnets, Polrings oder Maßstabs.

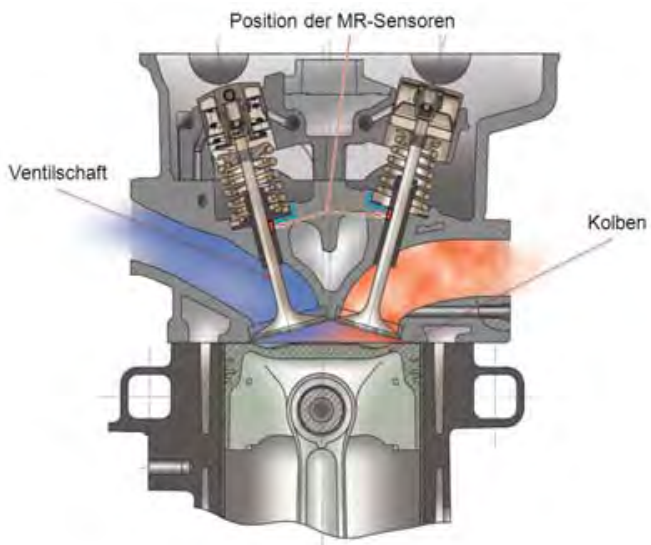

Abb. 14 Schnitt durch Zylinderkopf

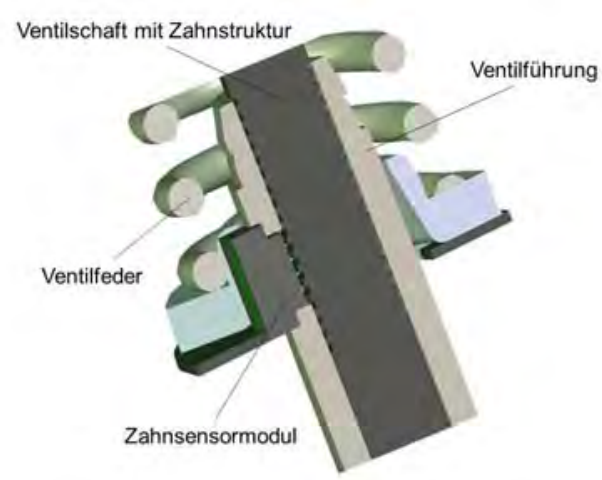

Abb. 15 Modifiziertes Zahnsensormodul für Ventilhubmessung

Dieses Prinzip wird auch für Anwendungen im Bereich Prüfstandstechnik bei der Motoren- und Fahrwerkentwicklung angewandt [10]. Beispielhaft ist die Anwendung des GLM Zahnsensormoduls für die Ventilhubmessung. Die Anforderungen an die Steuerung des Ladungswechsels bei Verbrennungsmotoren sind in den letzten Jahren stark gestiegen. Der Ventiltrieb steuert den Gaswechsel, von dem wiederum das Verbrennungsverhalten und die Leistungsfähigkeit eines Verbrennungsmotors abhängen. Der erforderliche Ventilhubverlauf muss deshalb über den gesamten Arbeitsbereich gewährleistet werden.

Bisher wurden Ventiltriebsdynamik-Messungen hauptsächlich an geschleppten Zylinderkopfattrappen mittels Laser-Doppler-Vibrometer durchgeführt. Um die Ventilbewegung direkt am Ventilteller messen zu können, müsste dabei die Brennraumdecke zugänglich gemacht werden. Viele Einflüsse aus dem realen Motorbetrieb bleiben dabei unberücksichtigt. Um ein genaueres Bild zu bekommen, muss die Ventiltriebuntersuchung im befeuerten Motorbetrieb durchgeführt werden. Abb. 14 zeigt wie GMR Zahnsensormodule eingesetzt werden, um solche Messungen am befeuerten Motor möglich zu machen. Die Ventile 
werden mit einer Zahnstruktur versehen, welche vom Zahnsensormodul gelesen werden kann. Die kompakten Abmessungen des Moduls ermöglichen den Einbau in kleinsten Bauräumen und die Sensormodule werden direkt in den Ventilführungen eingebaut (Abb. 15). Ausführliche Messreihen haben bestätigt, dass es keine wesentlichen Unterschiede gibt zu Ergebnissen, die mit einem Laser-Doppler-Vibrometer gemessen wurden, bei deutlich reduzierten Kosten. Der kompakte Aufbau macht sogar eine Ventiltriebsanalyse direkt auf einer Rennstrecke im Fahrzeug bei Extrembedingungen möglich.

\section{Ausblick}

Die oben beschriebenen Anwendungsbeispiele zeigen deutlich, wie magnetoresistive Sensorlösungen neue Möglichkeiten eröffnen. Anspruchsvolle Messaufgaben sind jetzt auch unter sehr schwierigen Einsatzbedingungen möglich. Es gibt jedoch weitere Anforderungen, die als Auslöser für weitere Entwicklungen dienen.

Es gibt neue Anwendungen, wo noch größere Luftspalten, noch höhere Drehzahlen (bis 1.000 .000 1/min) und die Abtastung von nicht-magnetischen (aber leitfähigen) Bauteilen gefordert sind. Auch hier gibt es die ersten MR-basierten Lösungsansätze.

\section{$8 \quad$ Literatur}

[1] Dixon, R.: Magnetic Sensors Head for the Big Time, Special Report on Magnetic Sensors, München: iSuppli, 2009

[2] Zwyssig, C., Duerr, M., Round, S., Kolar, J.: "An Ultra-High-Speed, 500,000 rpm, 1 kW Electrical Drive System", Proc. of $4^{\text {th }}$ Power Conversion Conference 2007, Nagoya, 2007.

[3] Celeroton AG: Data Sheet for Permanent-Magnet Machine CM-1-1000, Zürich, 2010.

[4] Korte, V., Hancock, D., Blaxill, H.: "Downsizing-Motor von Mahle als Technologiedemonstrator", MTZ 01/2008.

[5] Tumanski, S.: Thin Film Magnetoresistive Sensors, Bristol: Institute of Physics Publishing, 2001

[6] Ripka, P. (Ed.): Magnetic Sensors and Magnetometers, Boston: Artech House, 2001

[7] Paul, J. et al: Ressourceneffizienz am Beispiel magnetoresistiver Sensoren, Darmstadt: Mikrosystemtechnik Kongress 2011

[8] Slatter, R., Buß, R.: Neuartige magnetoresistive Sensorlösungen für rotatorische und lineare Antriebe, Würzburg: 8. ETG/GMM-Fachtagung Innovative Klein- und Mikroantriebstechnik, 2010

[9] Slatter, R.: Dynamic Position Measurement in High-Speed Applications using Magnetoresistive Sensors, Wetzlar: $11^{\text {th }}$ Symposium Magnetoresistive Sensors and Magnetic Systems, 2011

[10] Slatter, R.: Neuartige magnetoresistive Sensoren für Winkel- und Längenmessaufgaben im Automobil in Tille, T.: Sensoren im Automobil IV, Renningen, Expert Verlag, 2011 